\title{
Suicídio: aspectos epidemiológicos em Limeira e adjacências no período de 1998 a 2002
}

\author{
Suicide: epidemiological aspects in Limeira and \\ surroundings from 1998 to 2002
}

\author{
Makilim Nunes BAPTISTA \\ Amanda BORGES ${ }^{2}$
}

\begin{abstract}
Resumo
Para o presente estudo epidemiológico/documental foram selecionados os laudos de suicídios ( $\mathrm{n}=153$ ) no IML de Limeira (responsável por oito cidades da região), estado de São Paulo, Brasil, no período de janeiro de 1998 a dezembro de 2002. Os dados analisados foram os sociodemográficos: idade, sexo, estado civil, localidade (urbano/rural), além dos dados referentes aos métodos utilizados para a execução dos suicídios. Pode-se averiguar que os suicídios responderam por 6,2\% de todas as mortes no período citado, sendo mais prevalente em homens (82,0\%) e no meio urbano (88,9\%). A faixa etária mais atingida foi a de 21 a 40 anos para ambos os sexos, respondendo por $49,4 \%$ de toda a amostra e os métodos mais utilizados para os homens foram: enforcamento (56,0\%) e arma de fogo (24,0\%), e para as mulheres enforcamento (39,3\%) e intoxicação/veneno (21,4\%). A média geral de suicídios foi de 4,88/100.000 habitantes nas oito cidades pesquisadas no período. Os resultados são discutidos comparando-se os dados atuais com pesquisas epidemiológicas realizadas no Brasil.
\end{abstract}

Palavras-chave: epidemiologia; mortalidade; suicídio.

\begin{abstract}
This research analyzed 153 suicide reports, selected from 1998 to 2002 at the Legal Medical Institute in Limeira, SP (Brazil), whose assistance extends to eight cities nearby. Age, gender, marital status, localization (urban/rural) and main suicide methods were the studied data. According to the results, suicide represents $6.2 \%$ of all deaths. Also it is more common in males (82.0\%), and in urban areas (88.9\%). The age group from 21 to 40 is the most affected, in both sexes, and it represents $49.40 \%$ of the sample. The males' most used suicide methods are: hanging (56.0\%) and fire gun (24.0\%). Among women the most used methods are: hanging (39.3\%) and poisoning (21.4\%). The mortality rate was 4.88 suicides/100.000 inhabitants in this period. The results were compared to the data from Brazil epidemiological researches.
\end{abstract}

Key words: epidemiology; mortality; suicide.

$\operatorname{cor}$

1 Programa Pós-Graduação Stricto-Sensu em Psicologia, Universidade São Francisco. Rua Alexandre Rodrigues Barbosa, 45, 13251-900, Itatiba, SP, Brasil. Correspondência para/Correspondence to: M.N. BAPTISTA. E-mail: <makilim.baptista@saofrancisco.edu.br>

2 Psicóloga, consultório particular. Itatiba, SP, Brasil.

Agradecimentos: ao Dr. Eudes Freitas Aquino, médico legista do IML de Limeira, pela autorização e incentivo nesta pesquisa, ao Dr. Sydney Slly Urbach, delegado titular de Araras, pelo auxílio na solicitação da coleta de dados ao IML de Limeira, ao Dr. Wilson Schiavon de Abreu, médico legista do IML de Limeira, pela indicação de procedimentos na coleta de dados, à Carminda Souza Ferraz de Campos, encarregada do Setor Administrativo do IML de Limeira, pelo auxílio na coleta de dados, à Lourdes Aparecida Gambarato Olivatto, oficial administrativa do IML de Limeira e a Vicente Mettitier, auxiliar de necropsia do IML de Limeira. 
A questão do suicídio vem, há décadas, suscitando a curiosidade dos homens; diversas pesquisas vêm se concentrando em identificar quais as características associadas a indivíduos que tentam ou realizam o suicídio, bem como quais os fatores de risco relacionados a tais fenômenos (Diesederud, Royasamb, Ekeberg \& Kraft, 2001). O suicídio deve ser compreendido como um ato complexo, permeado por diversas variáveis, desde as questões genéticas até diversas variáveis psicológicas e socioculturais (Cassorla, 1992; Durkheim, 2000; Baptista, 2004)

Além das questões relacionadas ao sofrimento de quem tenta se suicidar ou mesmo dos parentes e amigos dos suicidas, outras avaliações devem ser consideradas. Hammond (2001) menciona os custos de ordem social e econômica que o suicídio representa para uma nação. Muitos países vêm despendendo somas significativas de dinheiro e treinamento de profissionais de saúde no intuito de diminuir a incidência do problema ou mesmo desenvolver programas preventivos nacionais. Dados da Organização Mundial de Saúde (2001) revelam que aproximadamente um milhão de pessoas cometem suicídio por ano em todo o mundo e entre 10 e 20 milhões de pessoas tentam se suicidar. O suicídio é uma das principais causas de morte de adultos jovens, situando-se entre as três principais na faixa etária entre 15 e 34 anos.

As tentativas bem como os suicídios estão ligados a uma série de fatores de risco que devem ser levados em consideração por profissionais de saúde, principalmente clínicos e equipes de ambulatórios e enfermarias, para que se possa diagnosticar precocemente indivíduos que tenham ideação suicida, e acompanhar, de forma mais focada, aqueles que já tentaram o suicídio. Algumas das variáveis associadas à tentativa e suicídio são depressão, alcoolismo, uso de z substâncias, idade, gênero, distresse, desemprego, perda 罢 de suporte social e condições médicas gerais (Lewis, Hawton \& Jones, 1997; Dhossche, Ulusarac \& Syed, 2001; Ikeda, Kresnow, Mercy, Powell, Simon, Potter, Durant \& $>\quad$ Swahn 2001). Outros fatores também podem estar o diretamente relacionados com a vulnerabilidade 令 psicológica para o suicídio, tais como impulsividade/ agressividade, desesperança, desengajamento social e atividade neuroumoral (Amaral, 1989; Weirauch,

426 Roy-Byrne, Katon \& Wilson, 2001).
Podem-se constatar diversas pesquisas epidemiológicas sobre ideação, tentativas e suicídios em diversos países desenvolvidos; no entanto, o Brasil ainda carece de mais pesquisas epidemiológicas sobre tal temática, bem como pesquisas que abarquem autópsias psicológicas em indivíduos que se suicidaram. Segundo Garcia-Perez (1998), autópsia psicológica é a caracterização retrospectiva da vida de uma pessoa já falecida, através de método indireto e inferencial, ou seja, através de relatos de familiares, colegas, companheiros de trabalho etc., e de revisão de documentos pessoais (notas, diários, cartas).

Por meio de um levantamento epidemiológico realizado por Silva, J., Silva, C., Silva, Jr., Silva, L. e Silva, D. (1999), em Salvador, no período de 1996 a 1997, detectaram-se que ser homem, não ter relacionamento afetivo estreito, estar na faixa etária entre 20 e 40 anos e ter apenas o curso primário são fatores de risco para o suicídio. Do contrário, ser do sexo feminino, estar casada, ter escolaridade elevada e mais de 61 anos seriam fatores de redução de risco para o suicídio.

Grossi, Marturano e Vansan (2000), a partir de uma revisão da literatura sobre a epidemiologia do suicídio de 1993 a 1997, comentam diversos fatores de risco associados com estado civil, ocupação, grupo étnico, imigração, condição socioeconômica e estado de saúde, assim como o aumento do risco em pessoas que possuem transtornos de personalidade/mental, experiências recentes de conflito e rompimento de relacionamentos, pobreza e presença de doença física grave. Vansan (1999), realizando um levantamento dos suicídios em Ribeirão Preto, SP, no período de 1990 a 1992, encontrou uma freqüência mais elevada no sexo masculino, sendo que na faixa etária entre 20 e 39 anos a prevalência de suicídios chega a ser quatro vezes maior em homens do que em mulheres. O mesmo autor ainda conclui que os fenômenos tentativa e suicídio são diferenciados com alguma área de sobreposição no que diz respeito aos dados sociodemográficos.

Souza, Minayo e Malaquias (2002) realizaram uma pesquisa documental por intermédio dos dados do sistema do Ministério da Saúde em capitais de nove regiões metropolitanas brasileiras (Belém, Fortaleza, Natal, Recife, Salvador, Belo Horizonte, Vitória, Rio de Janeiro, São Paulo, Curitiba e Porto Alegre), no período 
de 1979 a 1998. Encontrou-se, no conjunto das capitais, crescimento nas taxas de suicídio de 3,5 para 5/100 mil habitantes, na faixa etária de 15 a 24 anos, explicitando-se as taxas dos anos de $1979(3,5), 1985(3,4), 1990(4,0)$ e, finalmente, do ano de 1998, respondendo por 5,0 suicídios/100.000 habitantes na faixa etária especificada. Apesar de, no conjunto, os índices terem crescido, em algumas cidades constatou-se queda das taxas nos anos citados, tal como no Rio de Janeiro $(2,3 / 1,2 / 1,5 / 1,2 / 1,0)$, ou mesmo pouca variação nos índices, tal como ocorreu em Salvador $(0,6 / 0,3 / 0,5 / 0,6 / 0,4)$, ou até grandes variações, como ocorreu, por exemplo, em Porto Alegre (4,9/5,3/3,3/12,1/10,4), demonstrando ser um fenômeno com incidência instável no decorrer dos anos, em algumas regiões, talvez por sofrer a influência de diversos fatores, tais como os sociais, políticos e econômicos.

Meneghel, Victoria, Faria, Carvalho e Falk (2004), utilizando dados do Ministério da Saúde no período de 1980 a 1999 no estado do Rio Grande do Sul, encontraram aumento nas taxas de suicídios por 100 mil habitantes, tendo ido de nove nos anos oitenta para onze em 1999. Na cidade de Campinas, no período de 1976 a 2001, Marín-Leon e Barros (2003), utilizando o Banco de Dados de Óbitos de Campinas, encontraram taxas abaixo de 5/100 mil habitantes, no entanto as autoras não descartam a possibilidade dessas taxas serem sub-registradas.

É de suma importância identificar os dados sociodemográficos de suicidas, bem como avaliar a existência de padrões sociais, demográficos e seccionais desse fenômeno, já que se podem considerar insuficientes as pesquisas brasileiras na área. Para tal, o objetivo desta pesquisa foi avaliar algumas características demográficas e sociais referentes aos suicidas através de laudos do IML de Limeira, entre os anos de 1998 e 2002, bem como caracterizar os principais métodos utilizados nessa região.

\section{Método}

Tratou-se de uma pesquisa documental realizada no Instituto Médico Legal de Limeira, estado de São Paulo, no ano de 2003, através do levantamento dos laudos de suicídios no período de janeiro de 1998 a dezembro de 2002. Os dados tabulados se referiram à região, idade, sexo, raça, estado civil, zona urbana/rural e métodos mais utilizados.

O Instituto Médico Legal (IML) é um órgão que pertence à Secretaria de Segurança Pública do Estado, e é subordinado à Superintendência da Polícia TécnicoCientífica, sendo responsável por perícias no sentido de esclarecer mortes, análises tóxicas e dosagens alcoólicas. O Instituto Médico Legal da cidade de Limeira é responsável pelos dados estatísticos sobre perícias em medicina legal de oito cidades da região: Limeira, Araras, Iracemápolis, Cordeirópolis, Leme, Santa Cruz da Conceição, Pirassununga e Conchal.

A coleta se deu em quatro dias através da leitura das informações dos laudos arquivados em caixas. Foram avaliados um total de 2589 laudos (cerca de treze caixas com aproximadamente 200 laudos cada uma), com média de três a quatro caixas por dia, contendo os mais variados tipos de mortes. Inicialmente era lida exclusivamente a causa da morte nos laudos e, caso essa informação fosse identificada como suicídio, o laudo era separado e eram, então, coletados os demais dados sociodemográficos. Foram separados 153 casos de suicídios e os dados sociodemográficos referentes a eles foram compilados em uma planilha.

Para o cálculo do número de suicídios para cada 100 mil habitantes/ano, foi utilizada a variação do crescimento populacional por cidade/ano fornecido pelo Instituto Brasileiro de Geografia e Estatística (2005).

\section{Resultados}

Na análise geral, foram constatados 153 suicídios durante os 5 anos, sendo que na cidade de Santa Cruz da Conceição não houve nenhuma ocorrência de suicídio nesse período.

No ano de 1998, as mortes por suicídio responderam pelo quarto lugar em freqüência de todas as mortes ocorridas nas cidades citadas, ficando atrás dos homicídios, atropelamentos e mortes suspeitas, sendo que em 1999 o suicídio ocupou o sexto lugar. Em 2000 e 2001 as mortes por suicídio ocuparam o quinto lugar nas estatísticas gerais e, em 2002, o quarto lugar, precedidas pelos homicídios, acidentes de trânsito e mortes a esclarecer. 
A idade média da amostra foi de 39,3 $( \pm 15,4)$ anos, sendo que as mulheres possuíam menor média

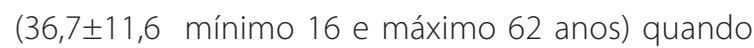
comparadas aos homens (39,9 \$16,1 mínimo 13 e máximo 86 anos). A maior prevalência de suicídios ocorreu na faixa etária de 21 a 40 anos, seguida pela meia idade, adolescência e terceira idade, em ambos os sexos (Tabela 1).

O cálculo da taxa de suicídio por 100 mil habitantes levando-se em consideração o gênero mostrou que os homens apresentaram uma média de 21,57/100 mil enquanto as mulheres tiveram um coeficiente de 5,38/100 mil habitantes nos cinco anos nos registros das cidades consultadas.

Na Tabela 2, observa-se que o suicídio foi mais freqüente em brancos e que $88,9 \%$ da amostra moravam no meio urbano. Em relação ao estado civil, observou-se que, em uma parcela significativa (45,8\%), esse dado não estava disponível nos laudos, o que restringe a idoneidade desses resultados.

Tabela 1. Suicídios por faixa etária e gênero.

\begin{tabular}{|c|c|c|c|c|c|c|}
\hline \multirow{2}{*}{ Faixa etária } & \multicolumn{2}{|c|}{ Masculino } & \multicolumn{2}{|c|}{ Feminino } & \multicolumn{2}{|c|}{ Total } \\
\hline & $n$ & $\%$ & $n$ & $\%$ & $n$ & $\%$ \\
\hline $13-20$ anos & 13 & 10,4 & 1 & 3,7 & 14 & 9,2 \\
\hline $21-40$ anos & 57 & 45,6 & 18 & 66,7 & 75 & 49,4 \\
\hline $41-65$ anos & 44 & 35,2 & 8 & 29,6 & 52 & 34,2 \\
\hline Acima de 65 anos & 11 & 8,8 & $0^{*}$ & $0,0^{*}$ & 11 & 7,2 \\
\hline Total & 125 & 100,0 & 27 & 100,0 & 152 & 100,0 \\
\hline
\end{tabular}

* Não houve discriminação da idade.

Tabela 2. Distribuição de suicídios por raça, estado civil e localidade.

\begin{tabular}{|c|c|c|c|}
\hline & & $n$ & $\%$ \\
\hline & \multicolumn{3}{|l|}{ Raça } \\
\hline & Branco & 131 & 85,6 \\
\hline \multirow{10}{*}{ 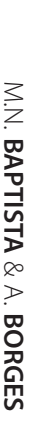 } & Pardo & 17 & 11,1 \\
\hline & Negro & 5 & 3,3 \\
\hline & \multicolumn{3}{|l|}{ Estado Civil } \\
\hline & Casado & 33 & 21,6 \\
\hline & Solteiro & 28 & 18,3 \\
\hline & Divorciado/Desquitado & 10 & 6,5 \\
\hline & Amasiado & 8 & 5,2 \\
\hline & Viúvo & 4 & 2,6 \\
\hline & Não consta & 70 & 45,8 \\
\hline & \multicolumn{3}{|l|}{ Localidade } \\
\hline 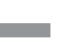 & Urbano & 136 & 88,9 \\
\hline & Rural & 6 & 3,9 \\
\hline 128 & Não consta & 11 & 7,2 \\
\hline
\end{tabular}

Os métodos mais utilizados pelos homens foram: enforcamento em 56\% dos casos; arma de fogo em 24\% e intoxicação (veneno) em 7,2\%, enquanto os métodos mais utilizados pelas mulheres foram o enforcamento (39,3\%), seguido de intoxicação/veneno $(21,4 \%)$ e queimadura (17,9\%) (Tabela 3$)$.

Em um comentário geral dos dados expressos naTabela 4 sobre o índice de suicídios para cada 100 mil habitantes, pode-se notar uma variação entre as cidades. Por exemplo, na cidade de Limeira a média geral de suicídios foi de 13,4 durante o período de cinco anos e 5,37 suicídios por 100 mil habitantes; em Araras, a média de suicídios foi de 6,2 e 5,97 suicídios por 100 mil habitantes; na cidade de Cordeirópolis a média foi de 0,6 e 3,40 suicídios por 100 mil habitantes; em Leme a média de 4,4 suicídios e 5,18 suicídios por 100 mil habitantes; em Pirassununga houve uma mesma média de suicídios $(4,4)$ mas a taxa por habitante foi de 6,64 por 100 mil habitantes devido à diferença populacional entre as cidades. Na cidade de Conchal a média de suicídios foi de 1,0 e 4,0 suicídios por 100 mil habitantes e, por último, na cidade de Iracemápolis a média de suicídios foi de 0,6 e 3,66 suicídios por 100 mil habitantes.

\section{Discussão}

Os suicídios vêm se apresentando como um problema de saúde pública em diversos países, sendo fundamental conhecer as variáveis implicadas em tal fenômeno para, assim, poder compreender melhor essa realidade. Estudos epidemiológicos podem também propiciar informações com o objetivo de criar

Tabela 3. Métodos de suicídios utilizados por gênero.

\begin{tabular}{lcccccc}
\hline & \multicolumn{2}{c}{ Sexo } & \multicolumn{2}{c}{ Masculino } & & \multicolumn{2}{c}{ Feminino } \\
\cline { 2 - 3 } \cline { 5 - 6 } Métodos & $n$ & $\%$ & & $n$ & $\%$ \\
\hline Enforcamento & 70 & 56,0 & & 11 & 39,3 \\
Arma de fogo & 30 & 24,0 & & 1 & 3,6 \\
Intoxicação/veneno & 9 & 7,2 & & 6 & 21,4 \\
Salto/pulo & 5 & 4,0 & & 2 & 7,1 \\
Queimadura & 5 & 4,0 & & 5 & 17,9 \\
Arma branca & 3 & & 2,4 & & 0 & 0 \\
Ingestão medicamentos & 2 & & 1,6 & & 1 & 3,6 \\
Desconhecido & 1 & 0,8 & & 2 & 7,1 \\
\hline Total & 125 & 100,0 & & 28 & 100,0 \\
\hline
\end{tabular}


Tabela 4. Distribuição do número de suicídios (total e por 100 mil habitantes) na região de Limeira de 1998 a 2002.

\begin{tabular}{|c|c|c|c|c|c|c|c|c|c|c|c|c|}
\hline \multirow{2}{*}{$\begin{array}{l}\text { Regiões de Limeira } \\
\text { (Suicídios por } 100 \text { mil habitantes) }\end{array}$} & \multicolumn{2}{|c|}{1998} & \multicolumn{2}{|c|}{1999} & \multicolumn{2}{|c|}{2000} & \multicolumn{2}{|c|}{2001} & \multicolumn{2}{|c|}{2002} & \multicolumn{2}{|c|}{ Média } \\
\hline & $n$ & $\%$ & $n$ & $\%$ & $n$ & $\%$ & $n$ & $\%$ & $n$ & $\%$ & $n$ & $\%$ \\
\hline Limeira & 17 & 7,06 & 11 & 4,48 & 9 & 3,60 & 15 & 5,91 & 15 & 5,82 & 13,40 & 5,37 \\
\hline Cordeirópolis & 1 & 6,04 & 0 & 0 & 0 & 0 & 1 & 5,52 & 1 & 5,40 & 0,60 & 3,40 \\
\hline Leme & 3 & 3,59 & 6 & 6,95 & 4 & 4,50 & 6 & 7,30 & 3 & 3,59 & 4,40 & 5,18 \\
\hline Pirassununga & 3 & 4,61 & 6 & 9,08 & 2 & 2,98 & 4 & 6,07 & 7 & 10,5 & 4,40 & 6,64 \\
\hline Conchal & 0 & 0 & 2 & 7,94 & 2 & 7,71 & 1 & 4,33 & 0 & 0 & 1,00 & 4,00 \\
\hline Iracemápolis & 0 & 0 & 2 & 0 & 0 & 0 & 0 & 0 & 3 & 18,34 & 0,60 & 3,66 \\
\hline
\end{tabular}

estratégias específicas no controle, bem como no desenvolvimento de centros especializados para a assistência de familiares e/ou indivíduos que tenham propensão para a ideação suicida ou tentativas anteriores. Como aponta Vansan (1999), os aspectos epidemiológicos e sociodemográficos de amostras que tentaram o suicídio e dos suicídios se mostram bem diferentes, principalmente em relação ao sexo e à distribuição etária, sugerindo serem a tentativa e o suicídio fenômenos que agrupam indivíduos com características peculiares.

Segundo Grossi e Vansan (2000), é necessário melhorar a qualidade dos dados disponíveis bem como desenvolver metodologias mais adequadas ao estudo desse tema. Como afirmam Botega e Rapeli (1995) e Silva et al. (1999), os dados oficiais sobre tentativas e suicídio no Brasil ainda carecem de um sistema confiável, o que pode distorcer a realidade desses fenômenos, inclusive no rigor do preenchimento de guias por parte dos IML. Por exemplo, no levantamento do estado civil dos prontuários da presente pesquisa, 45,7\% dos dados referentes ao estado civil não estavam preenchidos. Corrêa (1996) também aponta a falta de estatísticas confiáveis, pois um grande número de dados é classificado como "ignorado" ou mesmo as autoridades não têm condições de apurar detalhadamente as circunstâncias da morte.

No Brasil, Souza et al. (2002) encontraram, em uma pesquisa epidemiológica em diversas capitais do Brasil, o suicídio respondendo pela sexta causa de morte em populações jovens e urbanas ( 15 a 24 anos); na atual pesquisa, o suicídio variou entre o quarto e o sexto lugares em causa de mortes nas oito cidades pesquisadas da região de Limeira, o que leva a considerá-lo como um problema de saúde pública, sem contar os óbitos por esclarecer que podem deslocar ainda mais tais estatísticas, podendo o suicídio elevar posições no ranking das principais causas de morte.

O suicídio vem contribuindo sobremaneira para o total de mortes por causas externas registradas pelas estatísticas oficiais, como demonstram Pordeus, Fraga e Olinda (2002), que encontraram, no período de 1990 a 1998, entre todas as declarações de morte da Secretaria de Saúde do Estado do Ceará, 6,20\% de suicídios. Esse dado também confirma os dados da atual pesquisa que encontrou, em um período de cinco anos (1998-2002), uma taxa média de 6,21\%, observando-se uma variação anual de 3,94\% até 9,38\%; os anos de 2001 e 2002 foram os que apresentaram os maiores índices, ou seja, 8,51\% e 9,38\%, respectivamente.

Em relação à faixa etária, os resultados da presente pesquisa destacam o jovem adulto $(49,4 \%)$ com maior predominância na porcentagem por idade, seguido de sujeitos de meia idade (34,2\%); adolescentes (9,2\%) e terceira idade (7,2\%); tais dados são confirmados por algumas pesquisas e refutados por outras. Por exemplo, Lippi, Pereira, Soares e Camargos-Júnior (1990), por intermédio de uma pesquisa epidemiológica de suicídios no Estado de Minas Gerais, encontraram a faixa etária de 22 a 40 anos como a mais predominante (61,5\%), a segunda faixa etária acima de 40 anos com $21,0 \%$ do total e, de 13 a 21 anos (15,3\%) como a terceira faixa etária mais atingida. Apesar das diferenças na categorização das idades, os autores citados encontraram maiores porcentagens nas faixas da adolescência e jovens adultos e menores taxas em relação às idades acima de 40 anos, quando comparadas com os dados da atual pesquisa.

Pordeus et al. (2002) também encontraram as faixas etárias de adultos e adultos jovens como as mais 
pontuadas em suicídios, e Silva et al. (1999), por meio dos casos de suicídios registrados no IML da cidade de Salvador de janeiro de 1996 a dezembro de 1997, encontraram a faixa de 21 a 30 anos com a maior freqüência (24,89\%), seguida por 31 a 40 anos (24,45\%) e 16 a 20 anos (16,59\%), o que ratifica os dados da atual pesquisa, que encontrou $49,4 \%$ entre as idades de 21 a 40 anos, embora as outras faixas estejam dimensionadas de outra forma, inviabilizando a comparação mais direta.

Vansan (1999), em uma pesquisa realizada entre 1990 e 1992, no município de Ribeirão Preto, encontrou o equivalente a 40,6\% na faixa etária de 20 a 29 anos; na faixa etária de 30 a 39 anos, a porcentagem foi de 24,6\% e, na idade de 40 a 49 anos foi de 17,4\%. Esses dados são coerentes com a pesquisa de Lippi et al. (1990) e diferem dos dados atuais, pois tanto na pesquisa de Vansan quanto na de Lippi et al., a faixa etária de 20 a 40 anos é pontuada com uma média de $63 \%$ das amostras.

Quanto à variável sexo, pode-se observar uma concordância com grande parte das referências consultadas, mas com alguma variação, pois os dados da atual pesquisa demonstram uma maior porcentagem de homens quando comparada com os outros artigos. A relação de suicídio de homens e mulheres na amostra atual sugeriu 4,6:1 (homens $x$ mulheres), diferindo do índice encontrado por Vansan (1999) que foi de 2,8:1, mas se aproximando dos resultados encontrados por Meneguel et al. (2004), no Rio Grande do Sul, no período de 1980 a 1999, com uma proporção de cinco homens para cada mulher. O sexo masculino predominou na amostra atual com $82 \%$ das mortes por suicídio contra 73,9\% em Vansan (1999); 72,05\% em Silva et al. (1999) e 74,6\% em Grossi e Vansan (2002).

Quanto a esse fator, é sabido que o sexo masculino obtém maior sucesso nos suicídios do que o sexo feminino, apesar de as mulheres terem maior prevalência nas tentativas de suicídio. Isso se deve porque os homens utilizam métodos mais violentos e fatais do que as mulheres nas tentativas, como apontam Souza et al. (2002). Os coeficientes de mortalidade por sexo encontrados por Meneguel et al. (2004) no Rio Grande do Sul, que variaram para os homens de 14/100 mil em 1998 e 20,2/100 mil em 1999, são bem próximos dos encontrados na atual amostra (21,57/100 mil) na média dos cinco anos, o mesmo ocorrendo com as mulheres, que em 1998 (4,3/100 mil) e 1999 (4,1/100 mil) se aproximaram do 5,38/100 mil da atual pesquisa.

Em relação aos métodos de suicídio por gênero, os dados da atual pesquisa confirmam os encontrados por Marin-Leon e Barros (2003) em Campinas, no período de 1996 a 2001. Observou-se na pesquisa citada que os métodos mais prevalentes nos homens foram enforcamento, arma de fogo e envenenamento, o que também foi observado na atual pesquisa. Já para as mulheres, os dados encontrados demonstram que os métodos mais prevalentes foram enforcamento, envenenamento e queimadura, contrariamente aos descritos na pesquisa de comparação, sendo o envenenamento o método mais prevalente $(24,2 \%)$, seguido de enforcamento e armas de fogo, ambos com $21,2 \%$.

Dados epidemiológicos são muito úteis na caracterização dos fenômenos estudados. Especificamente no caso dos suicídios, quanto mais informações houver sobre tal caracterização, como, por exemplo, os dados socioeconômicos, dentre outros, maiores as chances dos profissionais de saúde e da população terem ferramentas no auxílio dessa problemática. Por exemplo, Barnes, Ikeda e Kresnow (2001) citam que grande parte dos suicidas tenta contato com familiares e/ou amigos, ou até mesmo com profissionais de saúde antes de cometerem o ato; assim as taxas de suicídios apresentadas no estudo atual poderiam ser menores se houvesse redes de apoio mais eficazes ou maior conhecimento das variáveis associadas à tentativa e suicídio no Brasil, já que o suicídio responde por uma parte significativa de todas as mortes ocorridas.

Sugere-se que as futuras pesquisas sobre suicídio no Brasil possam, além de ampliar os dados epidemiológicos brasileiros, também levantar dados específicos por intermédio de informações com familiares e amigos das pessoas que se suicidaram, a fim de se construir um mapeamento dos fatores de risco e situações que antecederam o ato suicida, para, em primeiro lugar, favorecer a compreensão do fenômeno no país, bem como favorecer o desenvolvimento de estratégias mais eficazes para a prevenção do suicídio.

As pesquisas baseadas em amostras epidemiológicas sobre as tentativas e os suicídios em diversas regiões brasileiras podem formar um corpo de conhecimento capaz de orientar as políticas públicas de saúde no desenvolvimento de programas de caráter 
primário e secundário na diminuição desses índices, sendo necessários mais estudos sobre o caráter epidemiológico do fenômeno, tanto com delineamentos quantitativos quanto qualitativos.

\section{Conclusão}

As características mais observadas neste estudo foram:

- o suicídio variou entre a quarta e a sexta causa mais freqüente de morte nas cidades pesquisadas, respondendo por 6,21\% do total de mortes;

- a taxa média geral de suicídios foi de 4,88/100 mil habitantes nas oito cidades pesquisadas, entre janeiro de 1998 e dezembro de 2002;

- o suicídio foi mais prevalente em homens (82\%) e a faixa etária mais atingida foi a de 21 a 40 anos em ambos os sexos;

- os métodos mais prevalentes de suicídio foram seqüencialmente o enforcamento, arma de fogo e intoxicação para os homens e enforcamento, intoxicação e queimadura para as mulheres;

- há dificuldades em avaliar dados mais precisos ou completos nos prontuários, tais como estado civil, grau de escolaridade, nível econômico, pré-existência de transtorno mental ou passagem em hospital psiquiátrico, ingestão de medicamentos psiquiátricos, dentre outros.

\section{Referências}

Amaral, M. (1989). Suicídio, depressão e atividade neurohumoral. Jornal Brasileiro de Psiquiatria, 38 (1), 31-35.

Baptista, M.N. (2004). Suicídio e depressão: atualizações. Rio de Janeiro: Guanabara-Koogan.

Barnes, L.S., Ikeda, R.M., \& Kresnow, M. (2001). Help-seeking Behavior Prior to Nearly Lethal Suicide Attempts. Suicide and Life-Threatening Behavior, 32 (suppl), 68-75.

Botega, N.J., \& Rapeli, C.B. (1998). Tentativa de Suicídio envolvendo risco de vida: internações em um hospital geral. Jornal Brasileiro de Psiquiatria, 44 (1), 157-162.

Cassorla, R.M.S. (1992). Oqueésuicídio. São Paulo: Brasiliense.

Correa, A.C. (1996). Depressão e Suicídio no idoso: Uma crucial questão de psicogeriatria. Jornal Brasileiro de Psiquiatria, 45 (3), 149-157.

Dhossche, D.M., Ulusarac, A., \& Syed, W. (2001). A retrospective study of general hospital patients who commit suicide shortly after being discharged from the hospital. Archives of International Medicine, 161 (7), 991-4.
Diesederud, G., Royasamb, E. Ekeberg, O. \& Kraft, P. (2001). Toward na Integrative Model of Suicide Attempt: A cognitive psychological approach. Suicide and Life-Threatening Behavior, 31 (2), 153-168.

Durkheim, E. (2000). O Suicídio. São Paulo: Martins Fontes.

Garcia-Perez, T. (1998). La autopsia en el suicidio. Medicina Legal Costa Rica, 15 (1-2), 22-24.

Grossi, R., Marturano, E.M. \& Vansan, G.A. (2000). Epidemiologia do suicídio - uma revisão da literatura. Jornal Brasileiro de Psiquiatria, 6 (1-2), 193-202.

Grossi, R., \& Vansan, G.A. (2002). Mortalidade por suicídio no município de Maringá (PR). Jornal Brasileiro de Psiquiatria, 51 (2), 101-111.

Hammond, R. (2001). Suicide Prevention: broadening the Field Toward a Public Health Approach. Suicide and Life-Threatening Behavior, 32 (suppl), 1.

Ikeda, R.M., Kresnow, M., Mercy, J.A., Powell, K.E., Simon, T.R., Potter, L.B., Durant, T.M., \& Swahn, M.H. (2001). Medical Conditions and Nearly Lethal Suicide Attempts. Suicide and Life-Threatening Behavior, 32 (suppl), 60-67.

Instituto Brasileiro de Geografia e Estatística. (2005, 1 março). Estatísticas do Século XX. Disponível em: www.ibge.org.br

Lewis, G., Hawton, K., \& Jones, P. (1997). Strategies for preventing suicide. British Journal of Psychiatry, 171, 351-354.

Lippi, J.R.S., Pereira, I.M, Soares, K.V.S., \& Camargos-Júnior, W. (1990). Suicídio na infância e adolescência. Jornal Brasileiro de Psiquiatria, 39 (4), 167-174.

Marín-Leon, L., \& Barros, M.B.A. (2003). Mortes por Suicidios: diferenças de gênero e nível sócio-econômico. Revista de Saúde Pública, 37 (3), 357-363.

Meneghel, S.N., Victoria, C.G., Faria, N.M.X., Carvalho, L.A., \& Falk, J.W. (2004). Características Epidemiológicas do Suicídio no Rio Grande do Sul. Revista de Saúde Pública, 38 (6), 804-810.

Organização Mundial de Saúde (2001). Relatório sobre a Saúde Mental no Mundo. Genebra: OMS.

Pordeus, A.M., Fraga, M.N., \& Olinda, Q.B. (2002). Suicídio no Ceará na década de 90. Revista Científica Ciências Saúde, $15(2), 16-22$

Silva, J.A., Silva, C.N., Silva Jr., Silva, L.N., \& Silva, D.N. (1999). Epidemiologia do suicídio na cidade de Salvador (BA). Revista Brasileira de Neurologia Psiquiátrica, 3 (1), 19-25.

Souza, E. R., Minayo, M.C., \& Malaquias, J.V. (2002). Suicídio de jovens nas principais capitais do Brasil. Caderno de Saúde Pública, 18 (3), 673-683.

Vansan, G.A. (1999). Aspectos epidemiológicos comparativos entre tentativas de suicídio e suicídios no município de Ribeirão Preto. Jornal Brasileiro de Psiquiatria, 48 (5), 209-215.

Weirauch, K.F., Roy-Byrne, P., Katon, W., \& Wilson, L. (2001). Stressful Life Events and Impulsiveness in Failed Suicide. Suicide and Life-Threatening Behavior, 31 (3), 311-319.

Recebido para publicação em 15 de abril de 2004 e aceito em 8 de agosto de 2005. 
